\title{
INVERSION OF CIRCULAR AVERAGES USING THE FUNK TRANSFORM
}

\author{
Can Evren Yarman, Birsen Yazıcı \\ Rensselaer Polytechnic Institute, \\ Electrical, Computer and System Engineering \\ Troy, NY
}

\begin{abstract}
In radar, when the wavelength of the transmitted electromagnetic wave is considerably larger than the dimension of the antenna, the received signal is modeled as the integral of the mean reflectivity function of the illuminated scene over the intersection of spheres centered at the transmitter location and the surface topography. When the surface topography is flat the received signal becomes integral of the mean reflectivity function over circles which is also referred to as circular averages of the mean reflectivity function. Thus, reconstruction of the ground reflectivity from synthetic aperture radar data requires inversion of the circular averages. Apart from radar, circular averages inversion also arises in thermo-acoustic tomography and sonar.

In this paper, we present a new inversion method for the circular averages that uses the relationship between the circular averages, hyperbolic x-ray and the Funk transforms[1]. The method is exact and numerically efficient as compared to standard filtered backprojection algorithms. Numerical simulations demonstrate that the approach is practical.
\end{abstract}

Index Terms - Synthetic aperture radar, circular averages, Funk transform, hyperbolic x-ray transform, hyperbolic geometry.

\section{INTRODUCTION}

In synthetic aperture radar (SAR), when the wavelength is considerably larger than the dimension of the antenna, the transmitted electromagnetic waves are almost spherical. For an aircraft cruising along a straight flight trajectory at a constant altitude with constant velocity, the received signal from a flat topography can be modeled by the circular averages of the ground reflectivity function [2, 3]. Here, the center of the circles corresponds to the projection of the flight path onto the ground as illustrated in Figure 1. While our work is primarily motivated by SAR, the problem of circular averages inversion also arises in other imaging modalities such as thermoacoustic tomography [4] and sonar [5].

Without loss of generality, assuming that the flight trajectory is along $x_{1}$-axis, the circular averages of the ground reflectivity $f$ is given by

$$
g(c, t)=\mathcal{R}_{C}[f](c, t)=\int_{0}^{2 \pi} f(c+t \cos \theta, t \sin \theta) d \theta
$$

where $c \in \mathbb{R}$ and $t \in \mathbb{R}^{+}$denote the center on $x_{1}$-axis and the radius of the circle, respectively. Thus, the reconstruction of the ground reflectivity function from the SAR data requires inversion of the circular averages.

This work was supported by Air Force Office of Scientific Research under the agreement FA9550-04-1-0223.

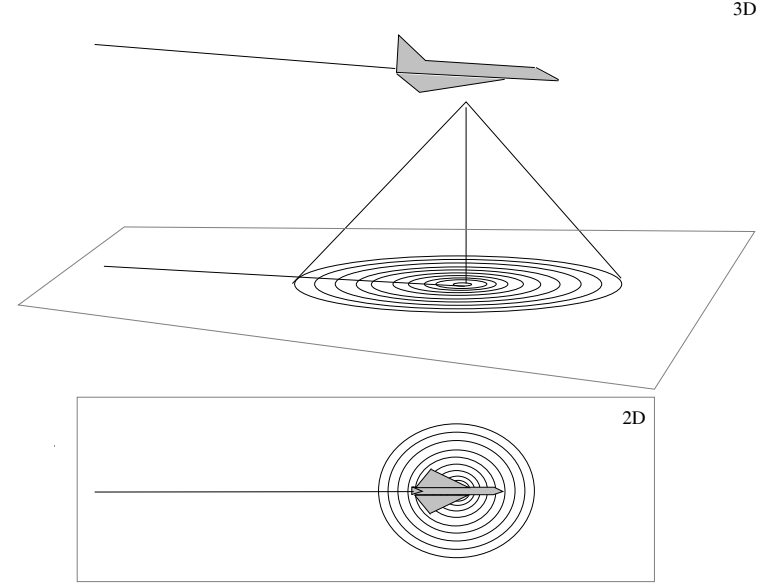

Fig. 1. 3D and 2D views of synthetic aperture radar geometry.

If a function $f$ is odd in its second variable, i.e. $f\left(x_{1}, x_{2}\right)=$ $-f\left(x_{1},-x_{2}\right)$, then its circular averages vanishes. The unique determination of a function $f$, which is symmetric with respect to $x_{1}$ axis, from its circular averages $g$ was proved by Courant and Hilbert [6]. As a consequence, for side looking radar, a function supported on the half-plane can be uniquely recovered from its integrals over semi-circles centered on the boundary of the half-plane, which, without loss of generality, we will refer to as the upper half-plane. For the rest of our discussion we will consider inversion of $g$ for functions that are symmetric with respect to $x_{1}$-axis. In this regard, we will not make any distinction between the circular averages of even functions and semi-circular averages of functions supported on the upper half-plane.

In [7], Lavrent'ev gave the characterizations of the range of $\mathcal{R}_{C}$ and an inversion method that requires infinite number of moments of $g$. In [8], Fawcett presented another inversion method using FourierHankel transform, where he showed that the Fourier-Hankel transform of $g$ is equivalent to the two-dimensional Fourier transform of $f$. In [3], Andersson gave an alternative proof of Fawcett's inversion method using the Fourier transform by treating $g$ as a radial function in terms of its second component

$$
g(c, \boldsymbol{t})=\int_{0}^{2 \pi} f(c+|\boldsymbol{t}| \cos \theta,|\boldsymbol{t}| \sin \theta) d \theta,
$$

where $t \in \mathbb{R}^{2}$. In [9], Nilsson reformulated Andersson's inversion formula in filtered backprojection form, which lead to a fast inversion algorithm. 
In this paper, we developed an alternative inversion method for the circular averages using the Funk transform.

Given a function $f$ defined on the unit sphere $S^{2}=\left\{x \in \mathbb{R}^{3}\right.$ : $|x|=1\}$, its Funk transform is defined by the integrals of $f$ along the great circles:

$$
\mathcal{M}[f](\boldsymbol{n})=\int_{S^{2}} f(\boldsymbol{x}) \delta(\boldsymbol{x} \cdot \boldsymbol{n}) d \boldsymbol{x}
$$

as illustrated in Figure 2, where $d \boldsymbol{x}$ is the normalized Haar measure on $S^{2}$.

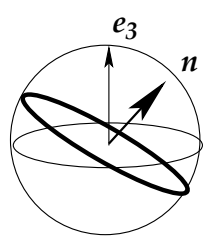

Fig. 2. Geometry of the Funk transform.

By (3), the Funk transform is even, i.e. $\mathcal{M}[f](-\boldsymbol{n})=\mathcal{M}[f](\boldsymbol{n})$, therefore only the even part of a function can be uniquely reconstructed from its Funk transform. In [10], Funk showed that even part of a function over $S^{2}$ can be uniquely determined from its Funk transform and presented two different inversion methods: The first method is an explicit way to compute the spherical harmonic decomposition of the function from the spherical harmonic decomposition of its Funk transform. The second method utilizes Abel's integral equation. We will focus on the first inversion method since it can be efficiently implemented using the fast Fourier transform algorithms over the rotation group and the sphere [11, 12].

In our study, we consider the circular averages in terms of the hyperbolic X-ray transform on the hyperbolic half-plane [13]. Then using the relationship between the hyperbolic x-ray transform on the hyperbolic half-plane and the Funk transform [1], we show that the inversion of circular averages is equivalent to the inversion of the Funk transform.

The organization of the paper is as follows. In Section 2, we present inversion of the Funk transform. In Section 3, we present the relationship between the circular averages and the Funk transform, and their inverse formulae. In Section 4, we give an inversion algorithm for the circular averages using the Funk transform. In Section 5 , we demonstrate the performance of the inversion method in numerical simulations. Finally, we conclude our discussion in Section 6.

\section{INVERSION OF THE FUNK TRANSFORM}

By (3), Funk transform is invariant with respect to rotations, i.e. $\mathcal{M}[f(Q \cdot)](\boldsymbol{\theta})=\mathcal{M}[f](Q \boldsymbol{\theta})$, where $Q \in S O(3)$ is a rotation matrix ${ }^{1}$. Since $S^{2}$ is a homogeneous space of $S O(3)$, any unit vector can be written as a product of a rotation matrix and the unit vector $\boldsymbol{e}_{\mathbf{3}}$. Using the homogeneity of $S^{2}$, we can express the Funk trans-

${ }^{1} S O(3)$ is a three parameter Lie group whose elements are 3 by 3 real orthogonal matrices with determinant equal to one. form as a convolution over the $3 \mathrm{D}$ rotation group $S O(3)$ [13]:

$$
\begin{aligned}
\mathcal{M}[f](\boldsymbol{n}) & =\int_{S^{2}} f(\boldsymbol{x}) \delta\left(\boldsymbol{x} \cdot \operatorname{Re}_{\mathbf{3}}\right) d \boldsymbol{x} \\
& =\int_{S O(3)} F(Z) \Lambda_{\mathcal{M}}\left(R^{-1} Z\right) d(Z) \\
& =\left(F *_{S O(3)} \Lambda_{\mathcal{M}}^{*}\right)(R),
\end{aligned}
$$

where $\boldsymbol{n}=\operatorname{Re}_{3}, F(Z)=f\left(Z \boldsymbol{e}_{3}\right), \Lambda_{\mathcal{M}}(Z)=\delta\left(Z \boldsymbol{e}_{3} \cdot \boldsymbol{e}_{3}\right)$ is the kernel of the Funk transform, $\delta\left(\boldsymbol{x} \cdot \boldsymbol{e}_{3}\right)$ being the Dirac measure of the equatorial circle and $d(Z)$ is the normalized invariant measure on $S O(3)$ which, using the Euler angles $\alpha, \gamma \in[0,2 \pi)$, and $\beta \in[0, \pi)$, is given by $d(Z(\alpha, \beta, \gamma))=d \alpha \sin \beta d \beta d \gamma$. By the $S^{2}$ invariance and convolution property of $S O(3)$-Fourier transform [14], the Funk transform can be diagonalized in the $S O(3)$-Fourier domain as follows:

$$
\widehat{\mathcal{M}}[f]_{m 0}^{2 l}=\widehat{F}_{m 0}^{2 l}{\overline{\widehat{\Lambda \mathcal{M}}^{2 l}}}^{2 l}
$$

where

$$
\begin{aligned}
\hat{F}_{m n}^{l}= & \frac{2 l+1}{8 \pi^{2}} \int_{0}^{2 \pi} \int_{0}^{\pi} \int_{0}^{2 \pi} F(\alpha, \beta, \gamma) \mathrm{e}^{\mathrm{i}(m \alpha+n \gamma)} \\
& \times \overline{d_{m n}^{l}(\beta)} d \alpha \sin \beta d \beta d \gamma, \quad|m|,|n| \leq l \in \mathbb{Z}^{+},
\end{aligned}
$$

and

$$
F(\alpha, \beta, \gamma)=\sum_{l \geq 0} \sum_{m=-l}^{l} \sum_{n=-l}^{l} \widehat{F}_{m n}^{l} \mathrm{e}^{-\mathrm{i}(m \alpha+n \gamma)} d_{m n}^{l}(\beta),
$$

are the $S O(3)$-Fourier and inverse $S O(3)$-Fourier transforms of $F$. Here $d_{m n}^{l}$ are the Wigner-D functions. Thus, for an even function $f$ on the unit sphere, inversion of the Funk transform can be implemented efficiently using fast $S O(3)$-Fourier algorithms $[11,12]$ in three steps by

$$
\mathcal{M}[f] \underset{\mathcal{F}_{S O(3)}}{\stackrel{\mathcal{M}[f]}{m 0}} l \stackrel{\left({\widehat{\Lambda_{\mathcal{M}}}}_{00}^{l}\right)^{-1}}{2} \widehat{f}_{m 0}^{l} \stackrel{\mathcal{F}_{S O(3)}^{-1}}{3} f .
$$

Due to the evenness of Funk transform, for the rest of the paper we will consider functions defined on the upper-hemisphere of $S^{2}$ that can be uniquely recovered from their Funk transforms.

\section{CIRCULAR AVERAGES AND FUNK TRANSFORM}

Let $S_{+}^{2}$ denote the upper hemisphere of $S^{2}, C_{+}=\left\{\boldsymbol{x} \in \mathbb{R}^{3}: x_{3}>\right.$ $\left.0, \arccos \left(x_{3} /|\boldsymbol{x}|\right)<\pi / 4\right\}$ and $H_{+}=\left\{\boldsymbol{y} \in \mathbb{R}^{3}: y_{1}=1, y_{3}>0\right\}$. Then the function $\Phi: C_{+} \cap S_{+}^{2} \rightarrow H_{+}$, defined by

$$
\Phi(\boldsymbol{x})=\left(1, \frac{2 x_{2}}{x_{1}+x_{3}}, \frac{2 \sqrt{x_{3}^{2}-\left(x_{1}^{2}+x_{2}^{2}\right)}}{x_{1}+x_{3}}\right)=\left(1, y_{2}, y_{3}\right),
$$

maps the intersection of the cone $C_{+}$and $S_{+}^{2}$ bijectively onto the upper half-plane $H_{+}$, while mapping the arcs of the semi-great circles of $S_{+}^{2}$ lying in $C_{+}$to the semi-circles centered on the boundary of $H_{+}[1]$ as illustracted in Figure 3. As a consequence, $\Phi^{-1}$ maps the semi-circles centered on the boundary of $H_{+}$onto the arcs of the semi-great circles of $S_{+}^{2}$ lying in $C_{+}$.

The projection $f(\Phi(\boldsymbol{x}))$ of a function $f$ on $H_{+}$back onto $C_{+} \cap$ $S_{+}^{2}$ can be treated as a function on $S^{2}$ supported on $C_{+} \cap S_{+}^{2}$. Integration of a function $f$ along the semi-circles centered on the boundary of $H_{+}$is known as the x-ray transform on the hyperbolic halfplane or hyperbolic x-ray transform on the upper half-plane [13]. 


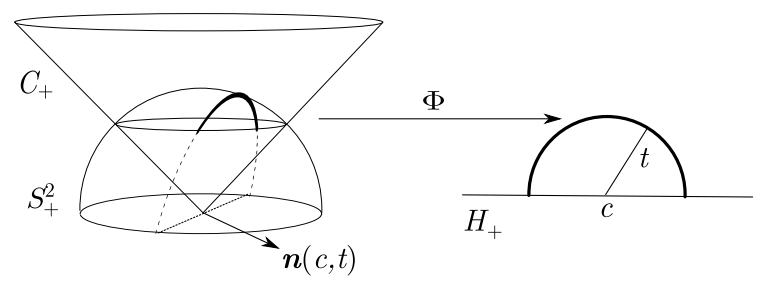

Fig. 3. Mapping $C_{+} \cap S_{+}^{2}$ to $H_{+}$by $\Phi$.

Thus computing the hyperbolic x-ray transform of $f$ on $H_{+}$, is equivalent to taking the Funk transform of the compactly supported function $f(\Phi(\boldsymbol{x})), \boldsymbol{x} \in C_{+} \cap S_{+}^{2}$, on $S^{2}$. In particular, given a great circle $Y$ perpendicular to the unit vector $\boldsymbol{n}$, we have [1]

$$
\int_{\Phi\left(Y \cap C_{+} \cap S_{+}^{2}\right)}^{f\left(y_{2}, y_{3}\right)} \sqrt{d s_{H_{+}}^{2}}=\int_{Y \cap C_{+} \cap S_{+}^{2}} \underset{\|(\boldsymbol{x}))}{f\left(\boldsymbol{x} \|_{H}^{2}\right.} \sqrt{d s_{C_{+} \cap S_{+}^{2}}^{2}},
$$

where $\|\boldsymbol{x}\|_{H}=\sqrt{x_{1}^{2}+x_{2}^{2}-x_{3}^{2}}$ is the hyperbolic metric, $d s_{H^{+}}^{2}$ and $d s_{C_{+} \cap S_{+}^{2}}^{2}$ are the associated Riemannian metrics on $H^{+}$and $C_{+} \cap S_{+}^{2}$ given by

$$
d s_{H_{+}}^{2}=\frac{d y_{2}^{2}+d y_{3}^{2}}{y_{3}^{2}}, \quad d s_{C_{+} \cap S_{+}^{2}}^{2}=d x_{1}^{2}+d x_{2}^{2}+d x_{3}^{2} .
$$

Equivalently, substituting (11) in (10) and replacing $f\left(y_{2}, y_{3}\right)$ with $y_{3} f\left(y_{2}, y_{3}\right)$, we can write (10) by

$$
t \mathcal{R}_{C}[f](c, t)=\|\boldsymbol{n}(c, t)\|_{H} \mathcal{M}\left[\frac{f(\Phi(\boldsymbol{x}))\left[\Phi(\boldsymbol{x}) \cdot \boldsymbol{e}_{\boldsymbol{3}}\right]}{\|\boldsymbol{x}\|_{H}^{2}}\right](\boldsymbol{n}(c, t)) .
$$

Thus, using the evenness of the circular averages and the Funk transform, the Funk transform can be inverted by the following formula:

$$
f(\Phi(\boldsymbol{x}))=\frac{\|\boldsymbol{n}\|_{H}^{2}}{\left[\Phi(\boldsymbol{x}) \cdot \boldsymbol{e}_{\mathbf{3}}\right]} \mathcal{M}^{-1}\left[\frac{t \mathcal{R}_{c} f(c, t)}{\|\boldsymbol{n}(c, t)\|_{H}}\right](\boldsymbol{x}) .
$$

\section{INVERSION OF CIRCULAR AVERAGES USING THE FUNK TRANSFORM}

In practical SAR data $g(c, t)$ is not available for all $c \in \mathbb{R}$ and $t \in \mathbb{R}^{+}$but $c \in\left[-c_{0}, c_{0}\right]$ and $t \in\left[0, t_{0}\right]$ for some $c_{0}, t_{0} \in \mathbb{R}^{+}$. Therefore, one needs to extrapolate the missing data for exact reconstruction. One of the advantage of the new method is that, since the Funk transform is defined on a compact surface like the sphere, extrapolation of the missing data, which is supported on unbounded domain, becomes an interpolation problem on the sphere that can be implemented in a numerically efficient manner.

We implemented inversion of the circular averages using the Funk transform in four steps as follows:

Step 1. Compute $\mathcal{M} G(\boldsymbol{n}(c, t))=t \mathcal{R}_{C} g(c, t) /\|\boldsymbol{n}(c, t)\|_{H}$;

Step 2. Interpolate $\mathcal{M} G(\boldsymbol{n})$ from $\mathcal{M} G(\boldsymbol{n}(c, t))$ for uniformly sampled $\boldsymbol{n} \in S^{2}$;

Step 3. Compute $G(\boldsymbol{x})$ by taking the inverse Funk transform of $\mathcal{M} G(\boldsymbol{n})$;

Step 4. Compute $f(\Phi(\boldsymbol{x}))=G(\boldsymbol{x})\|\boldsymbol{x}\|_{H}^{2} /\left[\Phi(\boldsymbol{x}) \cdot \boldsymbol{e}_{\mathbf{3}}\right]$.

\section{NUMERICAL SIMULATIONS}

In our numerical simulations, we used numerical phantoms to demonstrate the performance of the new inversion method. We form the numerical phantoms by linear transposition of circular disks. The phantom parameters (centers and radii of the disks) are given in $\mathrm{Ta}-$ ble 1 .

We compute the circular average of the phantoms analytically, and form the data by uniform sampling $(c, t) \in[-10,10] \times[0,6]$ by $201 \times 119$ pixels. The scene of interest is $\left(x_{1}, x_{2}\right) \in[-10,10] \times$ $[0,20]$ which we uniformly discretize by $201 \times 201$ pixels.

Following the inversion algorithm presented in Section (4), we present the numerical phantoms, their corresponding circular average data and reconstructed images in Figure 4. We also present the horizontal cross section of the point spread function for a disk centered at $\left(x_{0}, y_{0}\right)=(0,2)$ with radius .125 in Figure (5).

Our numerical reconstructions supports the applicability of the new method.

\begin{tabular}{ccc}
\hline Phantom & $\left(x_{0}, y_{0}\right)$ & $r$ \\
\hline 1 & $(0,2)$ & .125 \\
& $(-.5,2)$ & .125 \\
& $(.5,2)$ & .125 \\
2 & $(0,2)$ & .125 \\
& $(0,1.5)$ & .125 \\
& $(0,2.5)$ & .125 \\
3 & $(0,2)$ & .125 \\
& $(.5,1.5)$ & .125 \\
& $(-.5,2.5)$ & .125 \\
\hline
\end{tabular}

Table 1. Phantom parameters where $\left(x_{0}, y_{0}\right)$ denote centers of the disks and $r$ the corresponding radii.

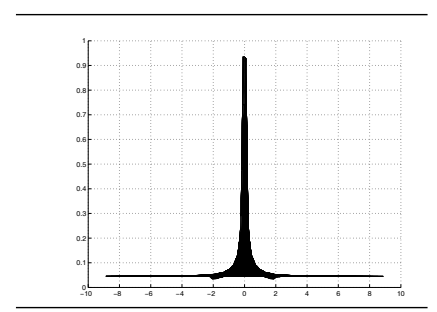

Fig. 5. Point spread function for a disk centered at $\left(x_{0}, y_{0}\right)=(0,2)$ with radius .125 .

The computational complexity of the new method is dominated by $S O(3)$-Fourier transform implementation required in the inversion of the Funk transform. Given a function defined on $S^{2}$, which is uniformly sampled at $2 B \times 2 B$ points in spherical coordinates, its $S O(3)$-Fourier coefficients can be computed in $\mathcal{O}\left(B^{2} \log ^{2} B\right)[12]$. In our simulations we chose $B=64$. For a scene sampled at $S \times S$ points, where $S=201$ in our numerical simulations, the computational complexity of the presented method is less than the standard filtered backprojection algorithms, which have computation complexity of $\mathcal{O}\left(S^{3}\right)$, yet comparable to or slower than the fast backprojection algorithms, which have computational complexity of $\mathcal{O}\left(S^{2} \log S\right)[9,15]$. 


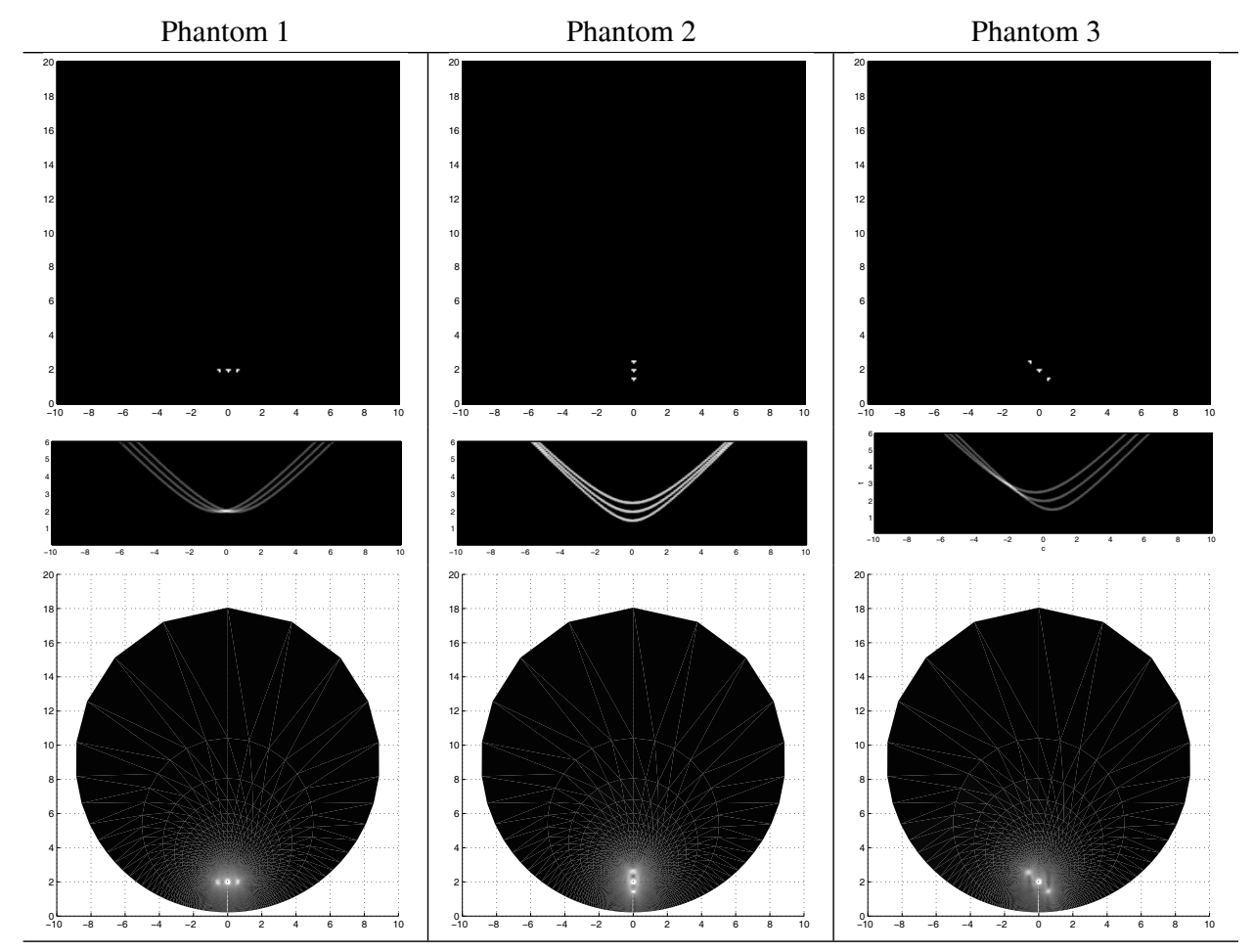

Fig. 4. (Top) Numerical phantoms, (middle) corresponding circular average data and (bottom) reconstructed images.

\section{CONCLUSION}

We have presented a new method for the inversion of the circular averages using the Funk transform. In our treatment we used the relationship between the hyperbolic geometry, circular averages and the Funk transform [1]. We considered the circular averages as the hyperbolic $\mathrm{x}$-ray transform and showed that inversion of circular averages is equivalent to inversion of the Funk transform for a class of compactly supported functions on the sphere, and developed a new inversion method. We demonstrated the performance of the proposed inversion method in numerical simulations.

\section{REFERENCES}

[1] C.E. Yarman, Inversion of Radon, exponential Radon, and Funk transforms based on harmonic analysis over groups, Ph.D. thesis, Rensselaer Polytechnic Institute, Troy, NY, 2006.

[2] H. Hellsten and L. E. Andersson, "An inverse method for the processing of synthetic aperture radar data," Inverse Problems, vol. 3, pp. 111-124, 1987.

[3] L. E. Andersson, "On determination of a function from spherical averages," SIAM J. Math. Anal., vol. 19, pp. 215-232, 1988.

[4] R. A. Kruger, D. R. Reinecke, and G. A. Kruger, "Thermoacoustic computed tomography," Med. Phys., vol. 26, pp. 1832$1837,1999$.

[5] A. K. Louis and E. T. Quinto, "Local tomographic methods in sonar," in Surveys on Solution Methods for Inverse Problems, D. Colton, H. W. Engl, A. K. Louis, J. R. McLaughlin, and W. Rundell, Eds., pp. 147-154. Springer-Verlag, Vienna, 2000.
[6] R. Courant and D. Hilbert, Methods of Mathematical Pyhscis, Vol. II, Partial Differential equations, John Wilter, New York, 1962.

[7] M. Lavrent'ev, V. Romanov, and V. Vasiliev, Multidimensional Inverse Problems for Differential Eqiuatons, vol. 167 of Lecture Notes in Mathematics, Springer-Verlag, Berlin, 1970.

[8] J.A. Fawcett, "Inversion of $n$-dimensional spherical averages," SIAM J. Appl. Math., vol. 45, no. 2, pp. 336-341, 1985.

[9] S. Nilsson, "Fast backprojection, technical report lith-isy-r1865," Tech. Rep., Linköping University: Department of Electrical Engineering, 1997.

[10] P. Funk, "Über Flächen mit lauter geschlossenen geodätischen Linien (on surfaces with louder closed geodesic lines)," Math. Ann., vol. 74, pp. 278-300, 1913.

[11] P. Kostelec and D. N. Rockmore, "FFTs on the rotation group," Santa Fe Institute Working Papers, Paper\#03-11-060, 2003.

[12] Jr. D. M. Healy, P. J. Kostelec, and D. Rockmore, “Towards safe and effective high-order Legendre transforms with applications to FFTs for the 2-sphere," Adv. Comput. Math., vol. 21, pp. 59-105, 2004.

[13] S. Helgason, Groups and Geometric Analysis: Integral Geometry Invariant Differential Operators, and Spherical Functions, American Mathematical Society, Providence, RI, 2000.

[14] N. Ja. Vilenkin, Special functions and the theory of representations, American Mathematical Society, Providence, RI, 1988.

[15] L.M.H. Ulander, H. Hellsten, and G. Stenström, "Syntheticaperture radar processing using fast factorized backprojection," IEEE Transactions on Aerospace and electronic systems, vol. 39, pp. 760-776, 2003. 